\title{
LncRNA ZFPM2-AS1 Promotes Metastasis and Epithelial-mesenchymal Transition of Hepatocellular Carcinoma via Targeting miR-3612/ADAM15 Axis
}

\author{
Nan Yang \\ Xi'an Jiaotong University \\ Tianxiang Chen \\ Xi'an Jiaotong University \\ Bowen Yao \\ Xi'an Jiaotong University \\ Liang Wang \\ Xi'an Jiaotong University \\ Runkun Liu \\ Xi'an Jiaotong University \\ Yongshen Niu \\ Xi'an Jiaotong University \\ Kangsheng Tu \\ Xi'an Jiaotong University \\ Zhikui Liu ( $\square$ liuzk0319@xjtufh.edu.cn ) \\ Xi'an Jiaotong University
}

\section{Research}

Keywords: Hepatocellular carcinoma, Long non-coding RNA, ZFPM2-AS1, miR-3612, ADAM15

Posted Date: July 17th, 2020

DOl: https://doi.org/10.21203/rs.3.rs-42530/v1

License: (c) (1) This work is licensed under a Creative Commons Attribution 4.0 International License.

Read Full License 


\section{Abstract}

Background: Long non-coding RNAs (IncRNAs) have obtained growing attention due to their potential effects as novel regulators in various tumors. This study aimed to investigate the expression and roles of IncRNA ZFPM2-AS1 in the progression of hepatocellular carcinoma (HCC).

Methods: Transwell was used to determine migration and invasion of HCC cells in vitro. The lung metastasis mouse model was established to detect tumor metastasis of HCC in vivo. The direct binding of miR-3612 to 3'UTR of DAM15 was confirmed by luciferase reporter assay. The expression of ZFPM2AS1 and miR-3612 in HCC specimens and cell lines were detected by real-time PCR. The correlation among ZFPM2-AS1 and miR-3612 were disclosed by a dual-luciferase reporter assay, RIP assay and biotin pull-down assay.

Results: In present study, we found that ZFPM2-AS1 was up-regulated in HCC tissues and cells and its upregulation was associated with TNM stage, vascular invasion, and poor prognosis of HCC patients. Functionally, gain- and loss-of-function experiments indicated that ZFPM2-AS1 promoted cell migration, invasion and EMT progress in vitro and in vivo. ZFPM2-AS1 could function as a competing endogenous RNA (ceRNA) by sponging miR-3612 in HCC cells. Mechanically, miR-3612 inhibited HCC metastasis and alternation of miR-3612 reversed the promotive effects of ZFPM2-AS1 on HCC cells. In addition, we confirmed that ADAM15 was a direct target of miR-3612 in HCC and mediated the biological effects of miR-3612 and ZFPM2-AS1 in HCC. Curcumin, an active derivative from turmeric, exerts its anticancer effects through ZFPM2-AS1/miR-3612/ADAM15 pathway. Our data identified ZFPM2-AS1 as a novel oncogenic IncRNA and correlated malignant clinical outcomes in HCC patients. Conclusions: ZFPM2-AS1 performed as oncogenic role via targeting miR-3612 and subsequently promoted ADAM15 expression in HCC. Our results revealed that ZFPM2-AS1 could be a potential prognostic biomarker and therapeutic target for HCC.

\section{Background}

Hepatocellular carcinoma (HCC) is one of the most frequently diagnosed cancers and a leading cause of cancer-related deaths worldwide, especially in South-Eastern Asia and Africa, causing nearly 745,000 deaths every year[1, 2]. Although the diagnosis and treatment technology of HCC have been improved in recent decades, including surgical resection, tumor ablation and transarterial chemoembolization, the clinical outcomes of HCC patients remain unsatisfactory because of the high rate of postoperative recurrence and metastasis[3]. Therefore, it is urgent to elucidate the molecular mechanisms underlying HCC progression and identify novel biomarkers for the diagnosis and treatment for HCC patients.

Long non-coding RNAs (IncRNAs) are a group of non-coding RNA molecules over 200 nucleotides in length, which is characterized by limited protein coding potential[4]. Abnormal expression of IncRNAs play a key role in cancer progression and carcinogenesis through various mechanisms, such as transcriptional regulation, chromatin remodeling, histone modification and mRNA splicing and stability[5- 
7]. Zinc finger protein multitype 2 antisense RNA 1 (ZFPM2-AS1) is a novel IncRNA which has not received much attention. Previous studies confirmed that IncRNA ZFPM2-AS1 promoted lung adenocarcinoma progression by interacting with UPF1 to destabilize ZFPM2[8]. LncRNA ZFPM2-AS1 enhances the malignancy of cervical cancer by functioning as a molecular sponge of miR-511-3p and consequently increasing FGFR2 expression[9]. ZFPM2-AS1 facilitated cell growth in esophageal squamous cell carcinoma via up-regulating TRAF4[10]. LncRNA ZFPM2-AS1 promotes the tumorigenesis of renal cell cancer via targeting miR-137[11]. However, the role of ZFPM2-AS1 in HCC remained largely unknown.

In this study, we confirmed for the first time that IncRNA ZFPM2-AS1 was up-regulated in HCC tissues and cell lines. High IncRNA ZFPM2-AS1 was associated with poor clinical features, including advanced TNM stage and vascular invasion, and worse prognostic survival. Gain- and loss-of-function experiment showed that ZFPM2-AS1 regulated migration, invasion, and epithelial-mesenchymal transition of HCC in vitro and in vivo. Furthermore, we explore whether ZFPM2-AS1 can regulate the expression of ADAM15 by regulating miR-3612 expression and affect the effects of HCC. Our results suggest that ZFPM2-AS1 exerts a critical role in HCC progression and might be a new molecular target for the treatment of HCC.

\section{Materials And Methods}

\section{HCC tissues and cell culture}

We collected HCC tissues and corresponding adjacent non-tumor tissues from our department. All patients received no therapy before surgery. All patients signed the informed consent. The patients' clinicopathological issues and demographic details were described in Table 1. The HCC cells (Hep3B, Bel7402, Huh7, MHCC-97H and HCCLM3) and normal immortalized liver cell L02 were obtained from Chinese Academy of Sciences (Shanghai, China). The cells were cultured in DMEM (Thermo Fisher, NY, USA) containing $10 \%$ fetal bovine serum (Gibco, Grandlsland, USA) in a humidified incubator at $37^{\circ} \mathrm{C}$ with $5 \% \mathrm{CO}_{2}$. 
Table 1: Clinical correlation of ZFPM2-AS1 expression in HCC $(n=101)$.

\begin{tabular}{|c|c|c|c|c|}
\hline \multirow[t]{2}{*}{ Clinical parameters } & \multirow{2}{*}{$\begin{array}{l}\text { Cases } \\
\text { (n) }\end{array}$} & \multicolumn{2}{|l|}{ Expression level } & \multirow{2}{*}{$\begin{array}{l}P \text { value } \\
\square^{*} p<0.05\end{array}$} \\
\hline & & ZFPM2-AS1 ${ }^{\text {high }}(\mathrm{n}=51)$ & ZFPM2-AS1 $1^{\text {low }}(n=50)$ & \\
\hline Age(years) & 65 & 32 & 33 & 0.733 \\
\hline$<60$ years & 36 & 19 & 17 & \\
\hline \multicolumn{5}{|l|}{$\geq 60$ years } \\
\hline \multicolumn{5}{|l|}{ Gender } \\
\hline Male & 80 & 38 & 42 & 0.240 \\
\hline Female & 21 & 13 & 8 & \\
\hline Tumor size (cm) & & & & 0.055 \\
\hline$\otimes 5 \mathrm{~cm}$ & 72 & 32 & 40 & \\
\hline$\geq 5 \mathrm{~cm}$ & 29 & 19 & 10 & \\
\hline Tumor number & & & & 0.199 \\
\hline solitary & 84 & 40 & 44 & \\
\hline multiple & 17 & 11 & 6 & \\
\hline Edmondson & & & & 0.444 \\
\hline$\nabla+\square$ & 23 & 10 & 13 & \\
\hline$\nabla+\square$ & 78 & 41 & 37 & \\
\hline TNM stage & & & & $0.003^{*}$ \\
\hline$\nabla+\square$ & 76 & 32 & 44 & \\
\hline$\nabla+\square$ & 25 & 19 & 6 & \\
\hline Vascular invasion & & & & $0.033^{*}$ \\
\hline Present & 16 & 12 & 4 & \\
\hline Absent & 85 & 39 & 46 & \\
\hline AFP & & & & 0.956 \\
\hline$\bigotimes 400 \mathrm{ng} / \mathrm{ml}$ & 24 & 12 & 12 & \\
\hline$\geq 400 \mathrm{ng} / \mathrm{ml}$ & 77 & 39 & 38 & \\
\hline HBsAg & & & & 0.527 \\
\hline positive & 91 & 45 & 46 & \\
\hline
\end{tabular}


HCC, hepatocellular carcinoma; AFP, alpha-fetoprotein; TNM, tumor-node-metastasis. *Statistically significant.

\section{qRT-PCR}

qRT-PCR was performed as reported previously[12-14]. TRIzol Reagent (Invitrogen) was used to extract total RNA from cells. PrimeScript RT Master Mix or TaqMan MicroRNA Reverse Transcription Kit (Applied Biosystems, Foster City, CA, USA) was used to reverse total RNA into cDNA. qRT-PCR was performed by SYBR Premix Ex Taq (Takara) and specifc primers. The results were calculated by the $2^{-\Delta \Delta C t}$ method. GAPDH and U6 was utilized as endogenous control.

\section{Western blot analysis}

Detailed assays were performed as previously reported[15-17]. Briefly, RIPA lysis buffer (Invitrogen) was used to extract total proteins. After examining the protein concentration by BCA Kit (Beyotime technology), 10\% SDS-PAGE was isolated the proteins and transferred to PVDF membranes. After blocking with $5 \%$ milk, membranes were cultured in primary antibodies (Cell signaling technology) and subsequently probed with secondary antibody. Then the signals were determined using chemiluminescence system (Thermo Fisher Scientific).

\section{Transwell assay}

The capacity of cells migration and invasion was evaluated via transwell assay. The upper chamber was pre-coated with BioCoat Matrigel (BD Biosciences, Franklin Lakes, NJ, USA) for invasion assay, whereas migration assay was not pre-coated with BioCoat Matrigel. Subsequently, $200 \mu$ cells were seeded into upper chamber which mixed with serum-free medium, $500 \mu$ l DMEM medium containing 10\% FBS was added into the lower chamber. After 24 hours incubation, cells on the upper chamber surface were removed using cotton swabs. Cells on the lower surface were fixed in $4 \%$ paraformaldehyde and stained with $0.5 \%$ crystal violet. Finally, NIS Elements image software (Nikon, Tokyo, Japan) was used to detect the number of migrating and invading cells.

\section{Luciferase reporter assay}

The experiments were performed similar as our previous studies[18]. The 3'UTR of ZFPM2-AS1 or ADAM15 wild type (wt) or mutant (mt) sequence was inserted in pmirGLO dual-luciferase vector (Genecopoeia, Guangzhou, China). Then the cells were co-transfected with miR-3612 or control mimics by Lipofectamine 2000 (Invitrogen) into HCC cells. Dual-Luciferase Reporter Assay System (Promega) was used to analyze the luciferase activity.

\section{RIP (RNA immunoprecipitation) assay}


We used the EZ-Magna RIP Kit (Millipore) to conduct the assays. The cells were lysed in RIP lysis buffer and incubated with magnetic beads of anti-Ago2 antibody (Millipore). Then the mix were incubated with proteinase $\mathrm{K}$ and the immunoprecipitated RNA was examined to real-time PCR analysis.

\section{In vivo metastasis assay}

4-6-week-old male BALB/c nude mice (Centre of Laboratory Animals, The Medical College of Xi'an Jiaotong University, Xi'an, China) were randomized into two groups $(n=5)$. Transfected cells $\left(1 \times 10^{6}\right)$ were injected into the tail veins for the establishment of the pulmonary metastatic model. Mice were sacrificed 3 weeks post-injection and examined microscopically by H\&E staining for the development of lung metastatic foci. Animals were housed in cages under standard conditions. All in vivo protocols were approved by the Institutional Animal Care and Use Committee of Xi'an Jiaotong University.

\section{Statistical analysis}

All data were expressed as the mean \pm SD and carried out by GraphPad Prism 6.0 (San Diego, USA). All experiments were performed at least three times. Survival analysis was plotted by Kaplan-Meier method and log-rank test. The relationship between PICSAR and clinical features was evaluated by Chi square test. The variance was analyzed by calculated by a Student's two-side t test. Difference with $\mathrm{P}<0.05$ was considered as significant.

\section{Results}

\section{Up-regulation of ZFPM2-AS1 in HCC was associated with poor prognosis}

The analysis of data from Gene Expression Profiling Interactive Analysis (GEPIA) (Fig.1A) and ENCORI Pan-Cancer Analysis platform (Fig.1B) described the expression of ZFPM2-AS1 was higher in the HCC tissues compared to non-tumor tissues. Moreover, the data from our cohort was consistent with this $(\mathrm{P}<0.05$, Fig. 1C). Moreover, our data showed that ZFPM2-AS1 was up-regulated in a group of HCC cells compared to normal hepatic cell L02 ( $<<0.05$, Fig. 1D). To clarify the clinical importance of ZFPM2-AS1 in HCC patients, we divided the patients into high or low subgroups according to the median value. As shown in Table 1, high ZFPM2-AS1 was associated with TNM stage $(P=0.003)$ and vascular invasion $(P=0.033)$. Kaplan-Meier survival analysis revealed that high ZFPM2-AS1 patients had a worse overall survival (OS) and disease-free survival (DFS) than patients with low ZFPM2-AS1 level ( $P=0.0006,0.0002$, respectively, Fig.1E). Moreover, the data from GEPIA (Fig.1F) and ENCORI (Fig.1G) also confirmed that ZFPM2-AS1 expression was dramatically correlated with OS and DFS. Taken together, these data suggest that ZFPM2-AS1 was up-regulated in HCC, and this up-regulation may play an important role in the progression of HCC.

\section{ZFPM2-AS1 promoted HCC cell migration, invasion and EMT in vitro and in vivo}


To further investigate the functional effects of ZFPM2-AS1 in HCC, we knockdown or overexpressed ZFPM2-AS1 with shRNA or plasmid in HCC cells $(P<0.05$, respectively, Fig.2A). As determined by Matrigelcoated (for invasion) and -uncoated (for migration) transwell assays, ZFPM2-AS1 overexpression significantly promoted the migration and invasion of Hep3B cells $(P<0.05$, Fig. 2B), whereas ZFPM2-AS1 knockdown obviously decreased the number of migrated and invaded HCCLM3 cells ( $P<0.05$, Fig. $2 \mathrm{C}$ ). EMT has been identified as a key process in the initiation of metastasis progression of HCC. To elucidate the potential role of ZFPM2-AS1 in HCC metastasis, we performed Western blot to confirm that ZFPM2AS1 overexpression decreased the epithelial marker E-cadherin and increased the mesenchymal marker $\mathrm{N}$-cadherin and Vimentin expression in Hep3B cells ( $\mathrm{P}<0.05$, Fig. 2D). By contrast, ZFPM2-AS1 knockdown showed opposite effects on EMT ( $P<0.05$, Fig. 2D). Moreover, we used tail vain injection to construct lung metastasis model. The data showed that ZFPM2-AS1 overexpression significantly promoted the number of lung metastasis of Hep3B cells whereas ZFPM2-AS1 knockdown reduce lung metastasis amounts ( $P<0.05$, Fig.2E). In conclusion, these data suggested that ZFPM2-AS1 could regulate HCC cell migration, invasion and EMT of HCC.

\section{ZFPM2-AS1 binds with and negatively regulates expression of miR-3612}

Previous studies confirmed that IncRNAs could sponge miRNAs by acting as competitive endogenous RNAs (ceRNAs) at post-transcriptional level[19]. To reveal the molecular mechanism of ZFPM2-AS1, we firstly searched location of ZFPM2-AS1 in IncLocator (http://www.csbio.sjtu.edu.cn/bioinf/IncLocator/) and found that ZFPM2-AS1 was located in the cytoplasm (Fig.3A).The subcellular fractionation assay showed similar results (Fig.3B). We searched 10 potential miRNAs sponged by ZFPM2-AS1 on ENCORI (http://www.sysu.edu.cn/403.html) and miR-3612 significantly inhibited the luciferase activity of ZFPM2AS1 vectors $(P<0.05$, Fig. $3 C$ ). Moreover, ZFPM2-AS1 regulated miR-3612 expression in HCC cells determined by qRT-PCR ( $P<0.05$, Fig. 3D). To test ZFPM2-AS1 exerts its function as a ceRNA, we performed anti-AGO2 RIP in HCC cells and the data showed that both ZFPM2-AS1 and miR-3612 were enriched in pulled down AGO2 protein (Fig.3E). Moreover, ZFPM2-AS1 was pulled down by biotin-labelled miR-3612, however, the ZFPM2-AS1 binding sites mutagenesis abolished the interaction between ZFPM2AS1 and miR-3612 ( $P<0.05$, Fig. 3F). In addition, miR-3612 was significantly down-regulated in HCC tissues compared to non-tumor tissues $(P<0.05$, Fig.3G). Pearson correlation analysis revealed that ZFPM2-AS1 expression was negatively associated with miR-3612 expression ( $r=-0.9322, P<0.01$, Fig. $3 \mathrm{H})$. Taken together, these data indicated that miR-3612 was a downstream target of ZFPM2-AS1 in HCC cells.

\section{miR-3612 mediates the effects of ZFPM2-AS1 on migration, invasion and EMT of HCC cells}

To explore the effects of miR-3612 in HCC, we confirmed that miR-3612 was down-regulated in HCC cells compared with L02 cells ( $P<0.05$, Fig.4A). We transfected HCCLM3 cells by lentivirus and knocked down miR-3612 in Hep3B cells with different endogenous miR-3612 expression to investigate the biological effects on HCC ( $P<0.05$, Fig.4B). MiR-3612 suppressed migration, invasion and EMT of HCC cells $(P<0.05$, Fig.4C-E). 
To investigate whether miR-3612 mediated the function of ZFPM2-AS1 in HCC cells, we co-transfected sh-ZFPM2-AS1 with miR-3612 inhibitors into HCC cells. The rescue experiments demonstrated that miR3612 overexpression vectors impaired the promotion effect of ZFPM2-AS1 on migration and invasion $(P<0.05$, Fig.4F-G). Moreover, miR-3612 inhibitors reversed the inhibitory effect of sh-ZFPM2-AS1 on migration and invasion ( $P<0.05$, Fig.4F-G). In conclusion, these data suggest that ZFPM2-AS1 promoted HCC migration and invasion partly by repressing miR-3612 function.

\section{miR-3612 directly targeted ADAM15 in HCC cells}

To elucidate the mechanisms of which miR-3612 exerted on HCC cells, we searched for the candidate targets by using bioinformatics (microRNA.org, TargetScan, and miRDB) and found ADAM15 3'UTR contains the binding sits of miR-3612 conserved putative (Fig.5A). To investigate the role of ADAM15 in HCC, we performed Western blot to show that ADAM15 was up-regulated in HCC tissues compared to that in normal tissues $(P<0.05$, Fig.5B), which was consistent with data from database UALCAN (http://ualcan.path.uab.edu/index.html; Fig.5C), GEPIA (http://gepia.cancer-pku.cn/; Fig.5C). Furthermore, data from UALCAN, GEPIA and Kaplan-Meier Plotter (Fig.5D) revealed that ADAM15 overexpression indicated poor survival of HCC patients. Moreover, ADAM15 was up-regulated in HCC cells compared to L02 cells $(P<0.05$, Fig.5E). ADAM15 promoted cell migration, invasion and EMT of HCC cells $(P<0.05$, Fig.5F-H). These data suggest that ADAM15 play an oncogene role in HCC.

To confirm ADAM15 was a target of miR-3612 in HCC cells, we used luciferase reporter assays to show that miR-3612 overexpression inhibited, while miR-3612 knockdown increased the luciferase activity of wild type (wt) ADAM15 3'UTR but not the mutant (mut) ADAM15 3'UTR ( $<<0.05$, Fig.6A). Furthermore, miR-3612 overexpression significantly inhibited the mRNA and protein of ADAM15 in HCCLM3 cells, while miR-3612 knockdown showed opposite effects in Hep3B cells ( $P<0.05$, Fig. 6B, $C)$. In HCC tissues, we confirmed an obvious inverse correlation between miR-3612 and ADAM15 mRNA ( $P<0.05$, Fig.6D). Thus, we conclude that miR-3612 directly targets ADAM15 in HCC cells.

\section{ADAM15 mediates the effects of ZFPM2-AS1 and miR-3612 on HCC cells}

To explore whether ADAM15 mediated the biological function of miR-3612 and ZFPM2-AS1 on HCC cells, ADAM15 was respectively restored in miR-3612-overexpressing or ZFPM2-AS1 knockdown HCCLM3 cells and inhibited by specific shRNA in miR-3612-suppressive or ZFPM2-AS1 overexpression Hep3B cells $(P<0.05$, Fig. 7A). ADAM15 restoration rescued the suppressive effects of miR-3612-overexpression or ZFPM2-AS1-knockdown HCCLM3 cells on cell migration, invasion and EMT progress ( $P<0.05$, Fig. 7B-D). Moreover, ADAM15 knockdown abolished the promotive effects of miR-3612 knockdown or ZFPM2-AS1 overexpression Hep3B cells ( $P<0.05$, Fig. 7B-D). These data confirmed that ADAM15 was functional mediators of ZFPM2-AS1/miR-3612 axis in HCC cells.

Curcumin attenuates HCC migration and invasion via ZFPM2-AS1/miR-3612/ADAM15 signaling pathway 
The above data clarify the molecular mechanisms of ZFPM2-AS1-mediated biological progress in HCC cells. To develop new agents to treat HCC, we found Curcumin, a yellow-colored dietary flavor from the plant (Curcuma longa), has been used in Chinses traditional medicine to treat various diseases, such as hepatic fibrosis, cancers[20-22]. Curcumin is a hydrophobic polyphenol derived from turmeric (Fig.8A). To confirm the inhibitory effects on HCC cells, we treated HCC cells with different concentrations of curcumin and cell viability was detected by MTT assays. As shown in Fig.8B, curcumin treatment significantly inhibited cell viability in a time- and dose-dependent manner. We choose $20 \mu \mathrm{M}$ for subsequent experiment. Curcumin obviously inhibited HCC cell migration, invasion and EMT progress $(\mathrm{P}<0.05$, Fig.8C-D). Moreover, curcumin significantly inhibited ZFPM2-AS1, ADAM15 expression while increased miR-3612 expression ( $P<0.05$, Fig.8E-G). Collectively, these data demonstrated that curcumin performed an anti-cancer effects on HCC cells.

\section{Discussion}

Accumulating evidence indicated that IncRNAs have the significant function in the carcinogenesis and the progression of malignancies during these years[23]. Therefore, it's crucial to investigate the regulatory characteristics of IncRNAs in the pathogenesis of HCC. In this study, we confirmed for the first time that IncRNA ZFPM2-AS1 was significantly up-regulated in HCC tissues and cell lines. Moreover, ZFPM2-AS1 expression was significantly associated with TNM stage and vascular invasion of HCC. These data suggest that ZFPM2-AS1 is an oncogene in HCC and plays a critical role in the progression of HCC.

Previous studies reported that IncRNA ZFPM2-AS1 was identified as a diagnostic and prognosis marker in cancers. ZFPM2-AS1 attenuated p53 pathway and promoted gastric carcinogenesis by stabilizing MIF[24]. ZFPM2-AS1 was involved in lung adenocarcinoma via miR-511-3p/AFF4 pathway[25]. ZFPM2AS1 promoted proliferation via miR-18b-5p/VMA21 axis in lung adenocarcinoma[26]. In this study, we demonstrated that ZFPM2-AS1 promoted cell migration, invasion and EMT progress by gain- and loss-of function experiment in vitro and in vivo. Previous studies reported that aberrant IncRNAs act as ceRNAs for miRNAs to modulate tumor development and progression[27]. In this study, we confirmed miR-3612 was remarkably down-regulated and negatively correlated with ZFPM2-AS1 in HCC tissues. Moreover, bioinformatics analysis, luciferase reporter assay, biotin pull-down assay and RIP assay all defined that miR-3612 was a target of ZFPM2-AS1 in HCC cells. In addition, we demonstrated that miR-3612 exerted its suppressive effects on migration and invasion of HCC cells. MiR-3612 mediated the biological function of ZFPM2-AS1 on HCC cells. Conclusively, ZFPM2-AS1 acted as miR-3612 sponge.

It has been confirmed that miRNAs could recognize and directly bind to their target mRNA 3' untranslated regions ( $3^{\prime}-U T R$ ) and consequently leading to the degradation or translation inhibition of mRNAs[28]. ADAM15, is a member of a family of catalytically active disintegrin membrane metalloproteinases that function as molecular signaling switches, shed membrane growth factors and cleave and inactivate cell adhesion molecules[29]. ADAM15 has been reported to be overexpressed in numerous malignancies including melanoma, breast cancer[30,31]. Here, we confirmed that ADAM15 is targeted by miR-3612 and overexpressed in HCC. Additionally, overexpressed ADAM15 recovered the effect of miR-3612 and 
ZFPM2-AS1 on HCC cell migration and invasion. Taken together, these results demonstrated that ZFPM2AS1 exert an oncogene role via miR-3612/ADAM15 axis in HCC.

Finally, we disclosed that curcumin, an active component of turmeric, displays various pharmacological activities[32]. The promising role of curcumin against different diseases is widely publicized[33].

Curcumin not only has attracted much attention for its anticancer properties, but also has been regarded to be safe in clinical trials owing to its low toxicity and good tolerance to human. Here, we demonstrated that curcumin inhibited HCC cell migration and invasion via regulating ZFPM2-AS1/miR-3612/ADAM15 axis.

In conclusion, we demonstrated that ZFPM2-AS1 was up-regulated in HCC, and could promote cell migration, invasion and EMT progression of HCC cells via ZFPM2-AS1/miR-3612/ADAM15 axis, which could be a valuable and promising therapeutic target for HCC.

\section{Conclusion}

To conclude, we reported for the first time that ZFPM2-AS1 was up-regulated in HCC tissues and cells. Its overexpression was associated with malignant clinical features and unfavorable prognosis. ZFPM2-AS1 facilitates HCC cell migration, invasion and EMT progress in vitro and in vivo. miR-3612 was identified as not only a target but also a functional mediator of ZFPM2-AS1 in HCC cells. ADAM15 was a direct target gene of miR-3612 and mediated its biological effects. Curcumin, as an inhibitor of HCC through regulating ZFPM2-AS1/miR-3612/ADAM15 pathway, provides novel mechanism for cancer therapy. In conclusion, ZFPM2-AS1/miR-3612/ADAM15 axis promoted cell migration and invasion of HCC. This finding will improve understanding of mechanism involved in cancer progression and provide novel targets for the molecular treatment of HCC.

\section{Abbreviations}

miRNAs, microRNAs; HCC, hepatocellular carcinoma; ADAM, A disintegrin and metalloprotease domain; IncRNA, Long non-coding RNA; qRT-PCR, real-time quantitative reverse transcription polymerase chain reaction; IHC, immunohistochemistry; IF, Immunofluorescence; RIP, RNA immunoprecipitation. 3'-UTR: 3'untranslated region; AFP: Alpha-fetoprotein; TNM: Tumor-node-metastasis; H\&E: Hematoxylin and eosin; EMT: Epithelial-mesenchymal transition

\section{Declarations}

Ethics approval and consent to participate: All procedures performed in studies involving human participants were in accordance with the ethical standards of the Research Ethics Committee of The First Affiliated Hospital of Xi'an Jiaotong University and with the 1964 Helsinki declaration and its later amendments. ALL written informed consent to participate in the study was obtained from HCC patients for samples to be collected from them. 
Consent for publication: Not applicable.

Availability of data and materials: All data generated or analyzed during this study are included either in this article.

Competing interests: The authors declare that they have no competing interests.

Funding: This study was supported by grants from Natural Science Basic Research Program of Shaanxi

(Program No.2020JQ-498; 2020JQ-496). Institutional Foundation of the First Affiliated Hospital of Xi'an Jiaotong University (2019QN-24).

Authors' contributions: ZKL conceived and designed the experiments; NY, TXC, BWY, LW and RKL performed the experiments; YSN and KST analyzed the data; KST contributed reagents/materials/analysis tools; NY and ZKL wrote the paper. All authors read and approved the final manuscript.

Acknowledgements: Not applicable

\section{References}

[1] A. Villanueva, Hepatocellular Carcinoma, The New England journal of medicine, 380 (2019) 14501462.

[2] F. Bray, J. Ferlay, I. Soerjomataram, R.L. Siegel, L.A. Torre, A. Jemal, Global cancer statistics 2018: GLOBOCAN estimates of incidence and mortality worldwide for 36 cancers in 185 countries, CA: a cancer journal for clinicians, 68 (2018) 394-424.

[3] L. Kulik, H.B. El-Serag, Epidemiology and Management of Hepatocellular Carcinoma, Gastroenterology, 156 (2019) 477-491 e471.

[4] J. Jarroux, A. Morillon, M. Pinskaya, History, Discovery, and Classification of IncRNAs, Advances in experimental medicine and biology, 1008 (2017) 1-46.

[5] S.U. Schmitz, P. Grote, B.G. Herrmann, Mechanisms of long noncoding RNA function in development and disease, Cellular and molecular life sciences : CMLS, 73 (2016) 2491-2509.

[6] Z. Liu, Y. Wang, L. Wang, B. Yao, L. Sun, R. Liu, T. Chen, Y. Niu, K. Tu, Q. Liu, Long non-coding RNA AGAP2-AS1, functioning as a competitive endogenous RNA, upregulates ANXA11 expression by sponging miR-16-5p and promotes proliferation and metastasis in hepatocellular carcinoma, Journal of experimental \& clinical cancer research : CR, 38 (2019) 194.

[7] L. Sun, L. Wang, T. Chen, Y. Shi, B. Yao, Z. Liu, Y. Wang, Q. Li, R. Liu, Y. Niu, K. Tu, Q. Liu, LncRNA RUNX1-IT1 which is downregulated by hypoxia-driven histone deacetylase 3 represses proliferation and cancer stem-like properties in hepatocellular carcinoma cells, Cell death \& disease, 11 (2020) 95.

[8] S. Han, D. Cao, J. Sha, X. Zhu, D. Chen, LncRNA ZFPM2-AS1 promotes lung adenocarcinoma progression by interacting with UPF1 to destabilize ZFPM2, Molecular oncology, 14 (2020) 1074-1088. 
[9] J. Dai, R. Wei, P. Zhang, P. Liu, Long Noncoding RNA ZFPM2-AS1 Enhances the Malignancy of Cervical Cancer by Functioning as a Molecular Sponge of microRNA-511-3p and Consequently Increasing FGFR2 Expression, Cancer management and research, 12 (2020) 567-580.

[10] G. Sun, C. Wu, ZFPM2-AS1 facilitates cell growth in esophageal squamous cell carcinoma via upregulating TRAF4, Bioscience reports, 40 (2020).

[11] J.G. Liu, H.B. Wang, G. Wan, M.Z. Yang, X.J. Jiang, J.Y. Yang, Long noncoding RNA ZFPM2-AS1 promotes the tumorigenesis of renal cell cancer via targeting miR-137, European review for medical and pharmacological sciences, 23 (2019) 5675-5681.

[12] Z. Liu, Y. Wang, C. Dou, L. Sun, Q. Li, L. Wang, Q. Xu, W. Yang, Q. Liu, K. Tu, MicroRNA-1468 promotes tumor progression by activating PPAR-gamma-mediated AKT signaling in human hepatocellular carcinoma, Journal of experimental \& clinical cancer research : CR, 37 (2018) 49.

[13] L. Wang, L. Sun, Y. Wang, B. Yao, R. Liu, T. Chen, K. Tu, Q. Liu, Z. Liu, miR-1204 promotes hepatocellular carcinoma progression through activating MAPK and c-Jun/AP1 signaling by targeting ZNF418, International journal of biological sciences, 15 (2019) 1514-1522.

[14] L. Sun, L. Wang, T. Chen, B. Yao, Y. Wang, Q. Li, W. Yang, Z. Liu, microRNA-1914, which is regulated by IncRNA DUXAP10, inhibits cell proliferation by targeting the GPR39-mediated PI3K/AKT/mTOR pathway in HCC, Journal of cellular and molecular medicine, 23 (2019) 8292-8304.

[15] Z. Liu, Y. Wang, C. Dou, M. Xu, L. Sun, L. Wang, B. Yao, Q. Li, W. Yang, K. Tu, Q. Liu, Hypoxia-induced up-regulation of VASP promotes invasiveness and metastasis of hepatocellular carcinoma, Theranostics, 8 (2018) 4649-4663.

[16] N. Yang, T. Chen, L. Wang, R. Liu, Y. Niu, L. Sun, B. Yao, Y. Wang, W. Yang, Q. Liu, K. Tu, Z. Liu, CXCR4 mediates matrix stiffness-induced downregulation of UBTD1 driving hepatocellular carcinoma progression via YAP signaling pathway, Theranostics, 10 (2020) 5790-5801.

[17] B. Yao, Y. Li, L. Wang, T. Chen, Y. Niu, Q. Liu, Z. Liu, MicroRNA-3194-3p inhibits metastasis and epithelial-mesenchymal transition of hepatocellular carcinoma by decreasing Wnt/beta-catenin signaling through targeting BCL9, Artificial cells, nanomedicine, and biotechnology, 47 (2019) 3885-3895.

[18] Y. Wang, L. Yang, T. Chen, X. Liu, Y. Guo, Q. Zhu, X. Tong, W. Yang, Q. Xu, D. Huang, K. Tu, A novel IncRNA MCM3AP-AS1 promotes the growth of hepatocellular carcinoma by targeting miR-194-5p/FOXA1 axis, Molecular cancer, 18 (2019) 28.

[19] R. Abdollahzadeh, A. Daraei, Y. Mansoori, M. Sepahvand, M.M. Amoli, J. Tavakkoly-Bazzaz, Competing endogenous RNA (ceRNA) cross talk and language in ceRNA regulatory networks: A new look at hallmarks of breast cancer, Journal of cellular physiology, 234 (2019) 10080-10100. 
[20] R. Zhao, S. Du, Y. Liu, C. Lv, Y. Song, X. Chen, B. Zhang, D. Li, S. Gao, W. Cui, M.V. Plikus, X. Hou, K. Wu, Z. Liu, Z. Liu, Y. Cong, Y. Li, Z. Yu, Mucoadhesive-to-penetrating controllable peptosomes-in-microspheres co-loaded with anti-miR-31 oligonucleotide and Curcumin for targeted colorectal cancer therapy, Theranostics, 10 (2020) 3594-3611.

[21] Y.H. Lee, N.Y. Song, J. Suh, D.H. Kim, W. Kim, J. Ann, J. Lee, J.H. Baek, H.K. Na, Y.J. Surh, Curcumin suppresses oncogenicity of human colon cancer cells by covalently modifying the cysteine 67 residue of SIRT1, Cancer letters, 431 (2018) 219-229.

[22] A. Eshaghian, A. Khodarahmi, F. Safari, F. Binesh, A. Moradi, Curcumin attenuates hepatic fibrosis and insulin resistance induced by bile duct ligation in rats, The British journal of nutrition, 120 (2018) 393-403.

[23] X. Chen, F.R. Tang, F. Arfuso, W.Q. Cai, Z. Ma, J. Yang, G. Sethi, The Emerging Role of Long NonCoding RNAs in the Metastasis of Hepatocellular Carcinoma, Biomolecules, 10 (2019).

[24] F. Kong, X. Deng, X. Kong, Y. Du, L. Li, H. Zhu, Y. Wang, D. Xie, S. Guha, Z. Li, M. Guan, K. Xie, Correction: ZFPM2-AS1, a novel IncRNA, attenuates the p53 pathway and promotes gastric carcinogenesis by stabilizing MIF, Oncogene, 37 (2018) 6010.

[25] J. Li, J. Ge, Y. Yang, B. Liu, M. Zheng, R. Shi, Long noncoding RNA ZFPM2-AS1 is involved in lung adenocarcinoma via miR-511-3p/AFF4 pathway, Journal of cellular biochemistry, 121 (2020) 2534-2542.

[26] M. Xue, W. Tao, S. Yu, Z. Yan, Q. Peng, F. Jiang, X. Gao, IncRNA ZFPM2-AS1 promotes proliferation via miR-18b-5p/VMA21 axis in lung adenocarcinoma, Journal of cellular biochemistry, 121 (2020) 313-321.

[27] Y. Fang, M.J. Fullwood, Roles, Functions, and Mechanisms of Long Non-coding RNAs in Cancer, Genomics, proteomics \& bioinformatics, 14 (2016) 42-54.

[28] K.C. Wang, H.Y. Chang, Molecular mechanisms of long noncoding RNAs, Molecular cell, 43 (2011) 904-914.

[29] A.P. Huovila, A.J. Turner, M. Pelto-Huikko, I. Karkkainen, R.M. Ortiz, Shedding light on ADAM metalloproteinases, Trends in biochemical sciences, 30 (2005) 413-422.

[30] J. Mattern, C.S. Roghi, M. Hurtz, V. Knauper, D.R. Edwards, Z. Poghosyan, ADAM15 mediates upregulation of Claudin-1 expression in breast cancer cells, Scientific reports, 9 (2019) 12540.

[31] C. Ungerer, K. Doberstein, C. Burger, K. Hardt, W.H. Boehncke, B. Bohm, J. Pfeilschifter, R. Dummer, D. Mihic-Probst, P. Gutwein, ADAM15 expression is downregulated in melanoma metastasis compared to primary melanoma, Biochemical and biophysical research communications, 401 (2010) 363-369.

[32] B. Ren, S. Luo, X. Tian, Z. Jiang, G. Zou, F. Xu, T. Yin, Y. Huang, J. Liu, Curcumin inhibits liver cancer by inhibiting DAMP molecule HSP70 and TLR4 signaling, Oncology reports, 40 (2018) 895-901. 
[33] T. Feng, Y. Wei, R.J. Lee, L. Zhao, Liposomal curcumin and its application in cancer, International journal of nanomedicine, 12 (2017) 6027-6044.

\section{Figures}
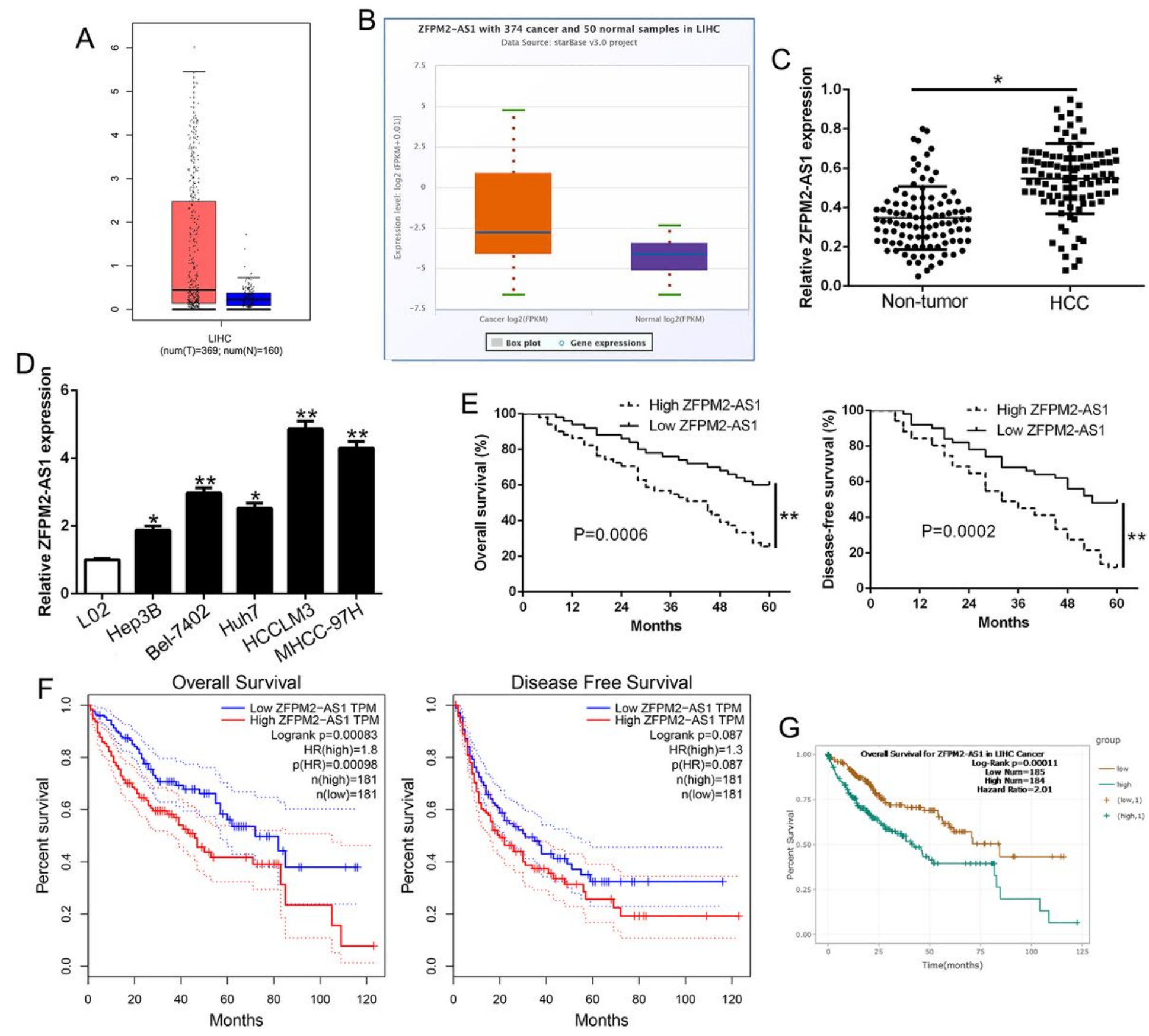

Figure 1

ZFPM2-AS1 is upregulated in HCC and is associated with poor prognosis. (A) GEPIA and (B) ENCORI platform database indicated that the expression of ZFPM2-AS1 was prominently higher in HCC tissues compared with normal liver tissues. (C) The real-time PCR data from our patient's cohort revealed that ZFPM2-AS1 was significantly up-regulated in HCC tissues than that in normal tissues. (D)The 
expressions of ZFPM2-AS1 in human normal hepatocyte cell line L02 and HCC cell lines were examined using qRT-PCR. (E) Kaplan-Meier survival curves of overall survival (OS) and disease-free survival (DFS) in our patients' cohort. (F) GEPIA and (G) ENCORI platform database demonstrated that high ZFPM2-AS1 expression also indicated poor survival of HCC patients. *P $<0.05$, **P $<0.01$.
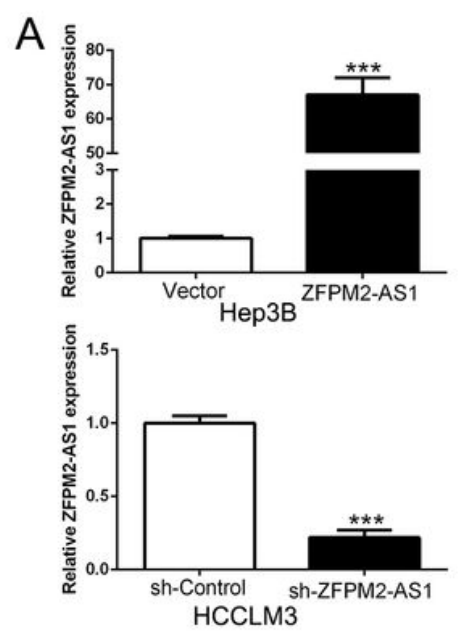

C

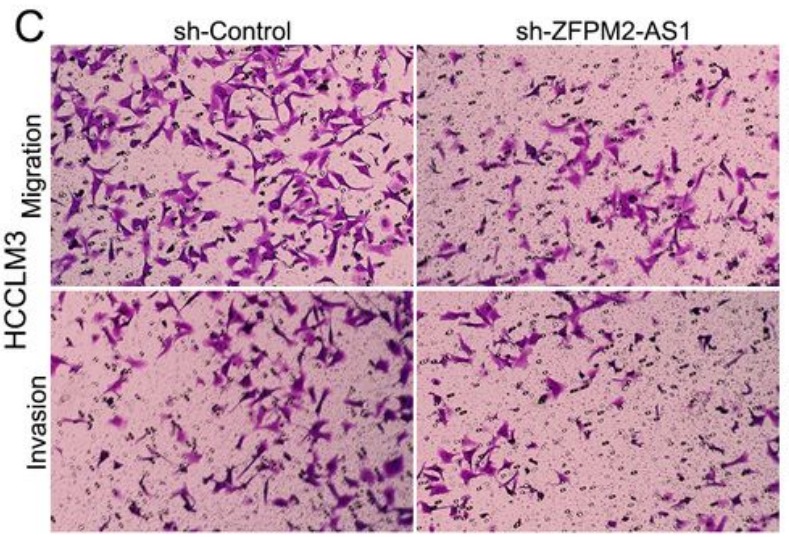

E

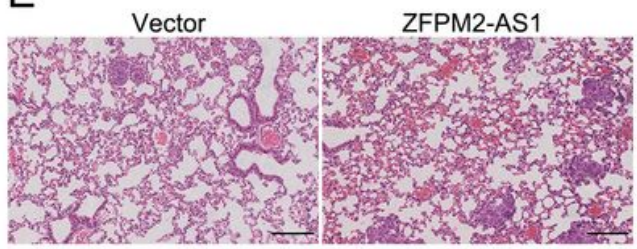

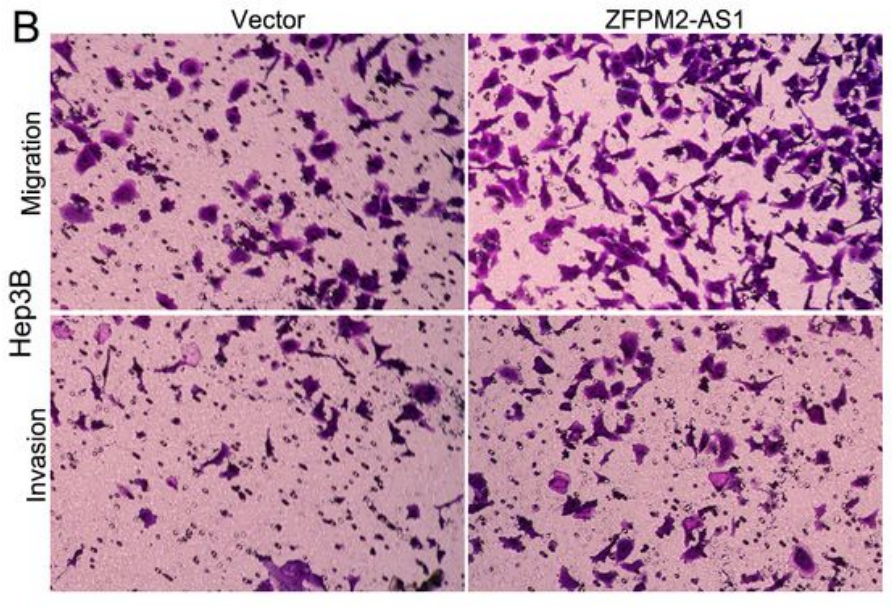
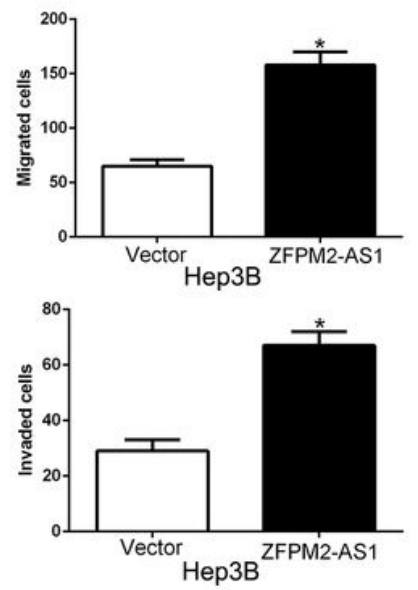
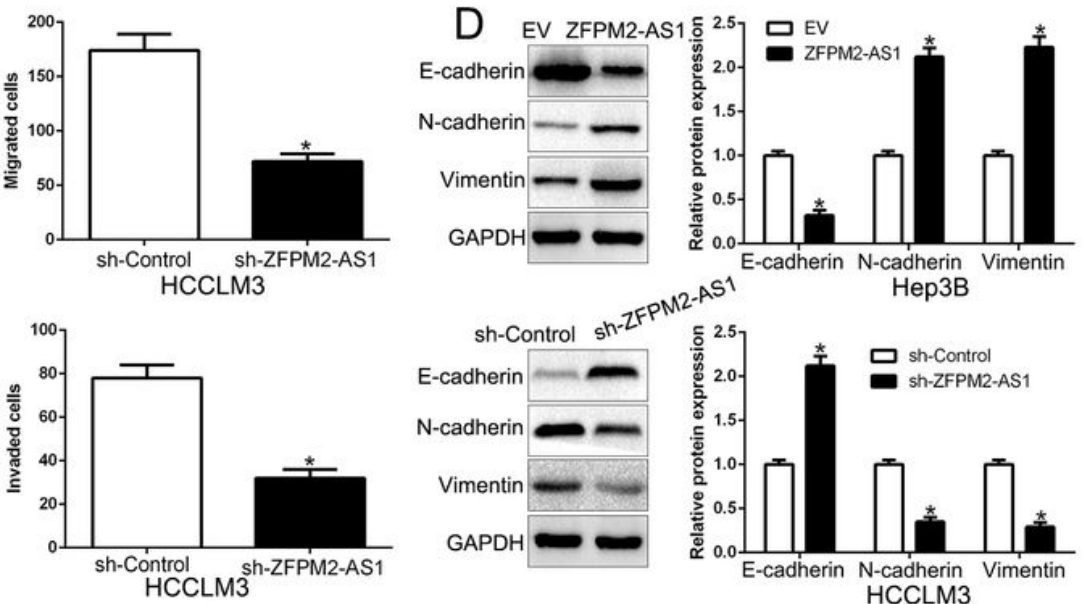
sh-ZFPM2-AS1

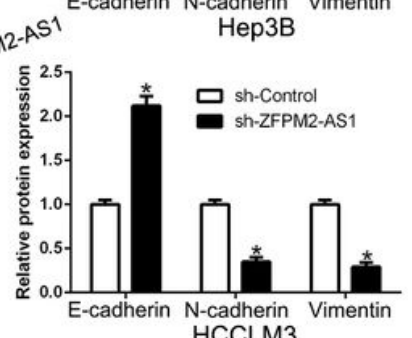

HCCLM3
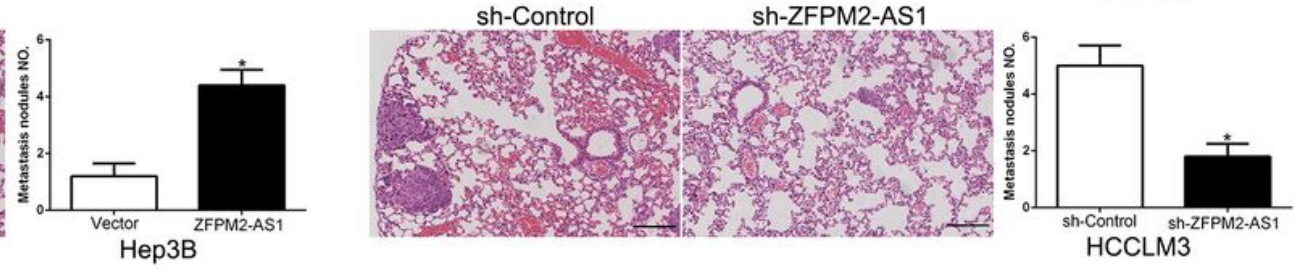

Figure 2

ZFPM2-AS1 promoted HCC cell migration, invasion and EMT in vitro and in vivo. (A) Hep3B and HCCLM3 cells that were transfected with corresponding vectors were subjected to qRT-PCR for ZFPM2-AS1 expression. (B) Overexpression of ZFPM2-AS1 promoted migration and invasion of Hep3B cells, while down-regulation of ZFPM2-AS1 inhibited migration and invasion in HCCLM3 cells (C). (D) Western blot analysis of E-cadherin, N-cadherin and Vimentin expression in the presence and absence of ZFPM2-AS1. (E) Representative HE staining of lung metastasis of Hep3B or HCCLM3 transfected with respective ZFPM2-AS1 vector. $n=$ three independent experiments. ${ }^{*} \mathrm{P}<0.05,{ }^{\star} * \star \mathrm{P}<0.001$. 

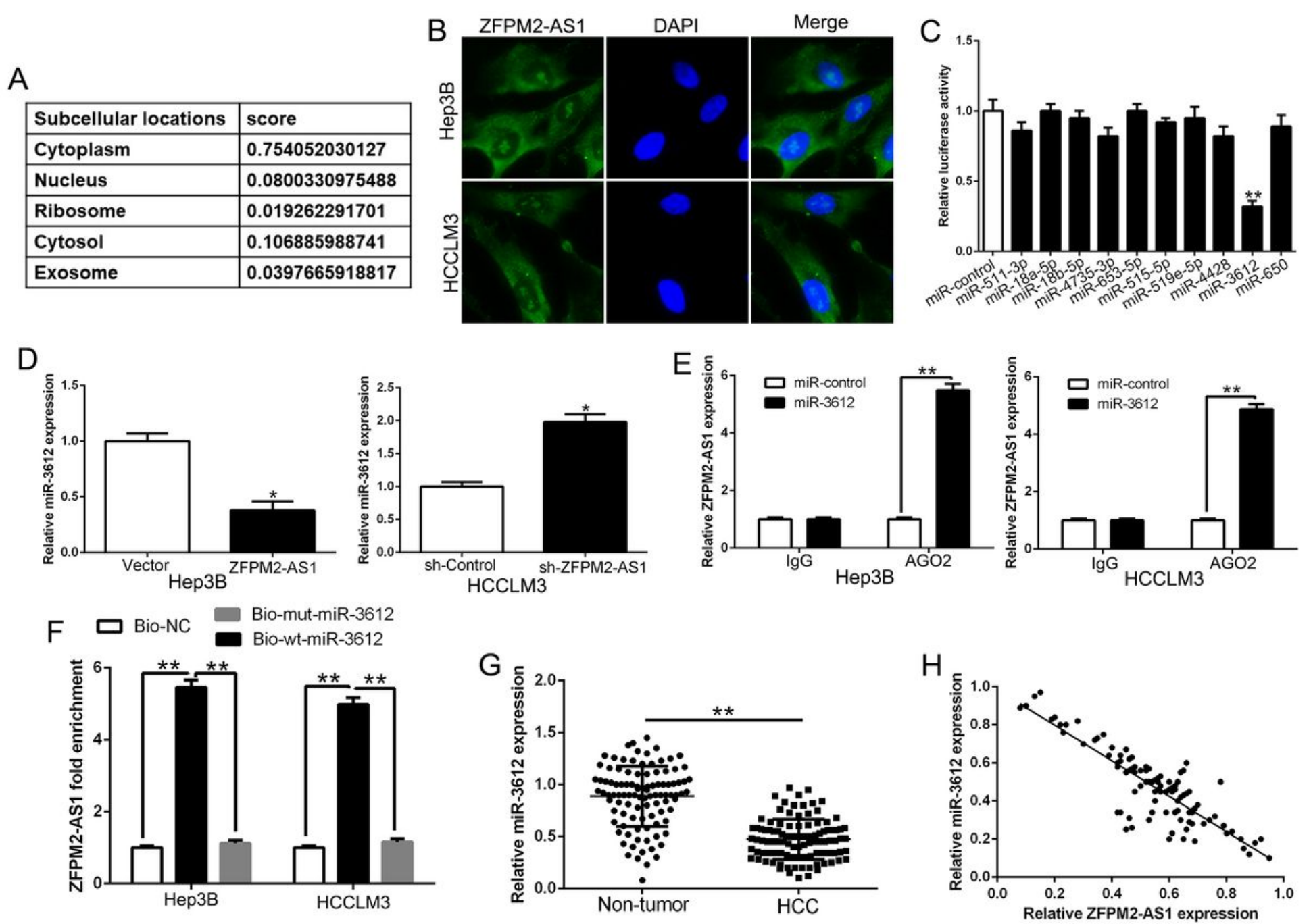

Figure 3

ZFPM2-AS1 binds with and negatively regulates expression of miR-3612. (A) The subcellular distribution of ZFPM2-AS1 from IncLocator database. (B) FISH assay was conducted to determine ZFPM2-AS1 localization. (C) Luciferase reporter assay was carried out to validate the interaction between ZFPM2-AS1 and predicted miRNAs. (D) The expression of miR-3612 was negatively regulated by ZFPM2-AS1. (E) The anti-Ago2 RIP assay with miR-3612 mimics showed that both miR-3612 and ZFPM2-AS1 were enriched in Ago2 precipitate compared to IgG. (F) ZFPM2-AS1 was highly enriched in the sample pulled down by biotinylated wt miR-3612 rather than mut miR-3612. (G) The expression of miR-3612 in tumor tissues was significantly lower than that in adjacent non-tumor tissues. $(\mathrm{H})$ Pearson correlation analysis revealed that there existed a negative association between miR-3612 and ZFPM2-AS1 in HCC tissues. $n=$ three independent experiments. ${ }^{*} \mathrm{P}<0.05$, ${ }^{\star *} \mathrm{P}<0.01$. 

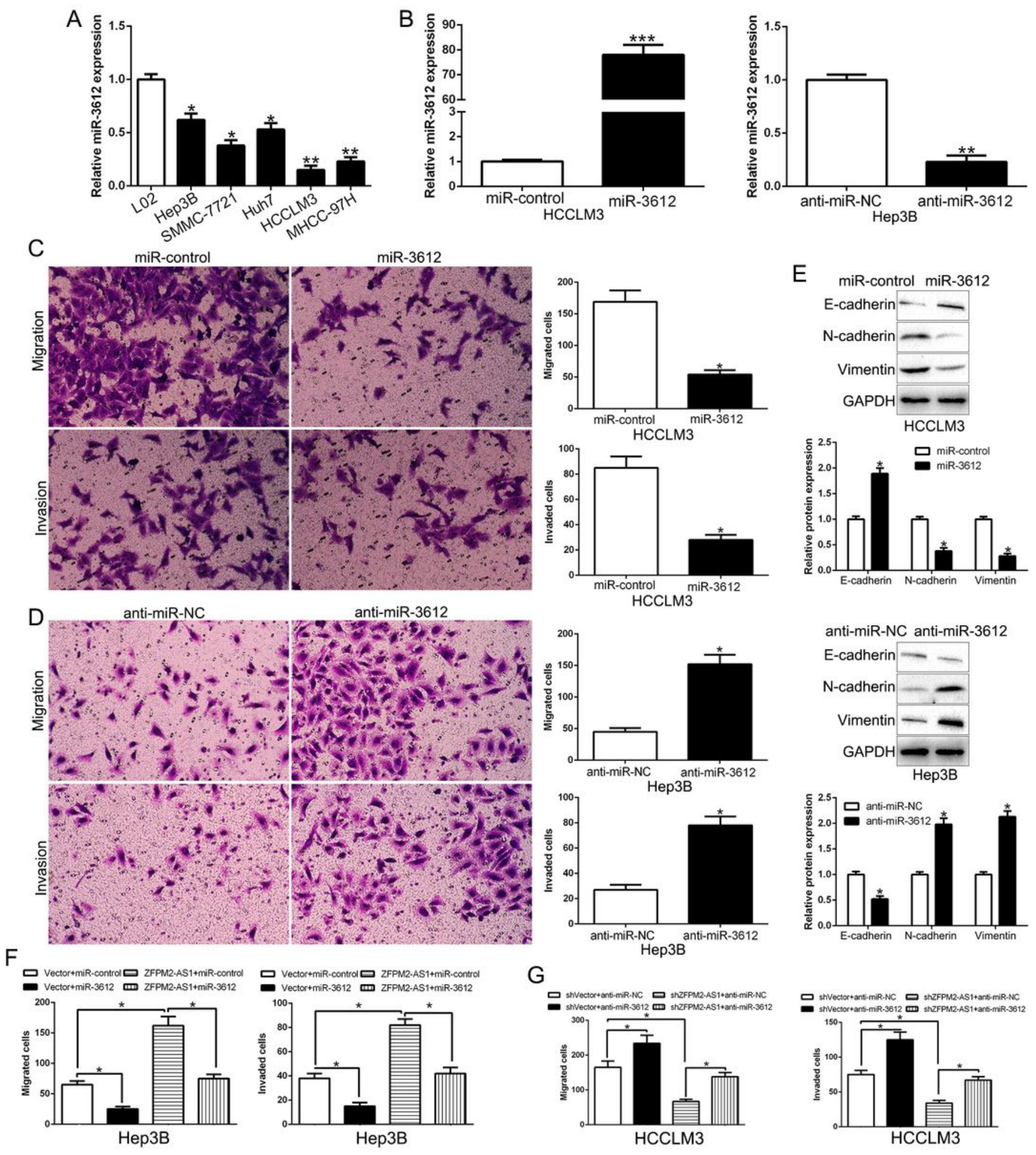

Figure 4

miR-3612 mediates the effects of ZFPM2-AS1 on migration, invasion and EMT of HCC cells. (A) The expressions of miR-3612 in human normal hepatocyte cell line L02 and HCC cell lines were examined using qRT-PCR. (B) Hep3B and HCCLM3 cells that were transfected with corresponding miRNA vectors were subjected to qRT-PCR for miR-3612 expression. Overexpression of miR-3612 inhibited cell migration and invasion (C) in HCCLM3 cells, while down-regulation of miR-3612 promoted cell migration and 
invasion in Hep3B cells (D). (E) Western blot analysis of EMT-associated factors expression in the presence and absence of miR-3612. (F) miR-3612 restoration abrogated the effects of ZFPM2-AS1 overexpression on cell migration and invasion of Hep3B cells. (G) miR-3612 knockdown reversed the suppressive effects of ZFPM2-AS1 knockdown in HCCLM3 cells. $n=$ three independent experiments. $* \mathrm{P}<0.05$.

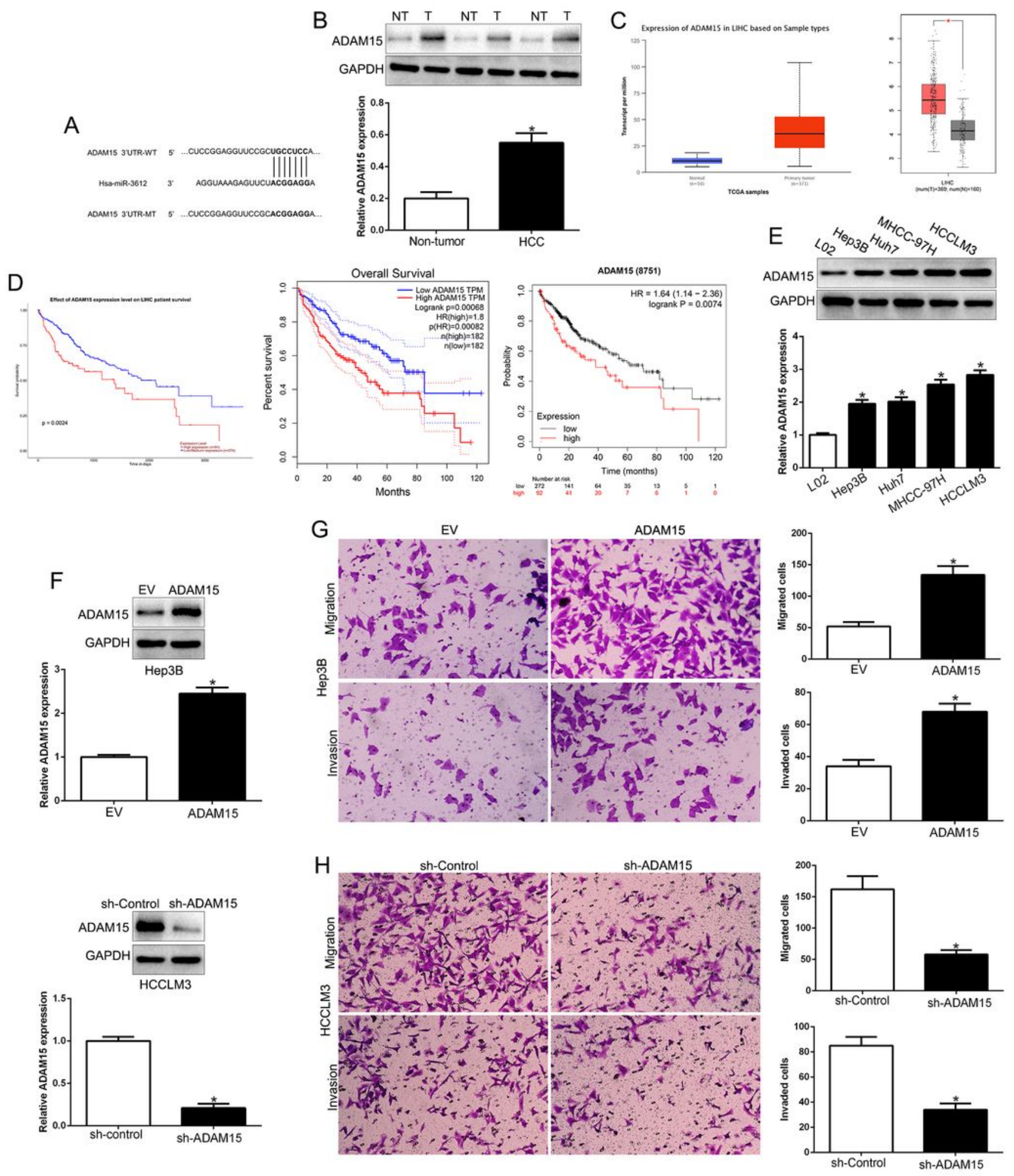

Figure 5 
miR-3612 directly targeted ADAM15 in HCC cells. (A) miR-3612 and its putative binding sequence in the 3'-UTR of ADAM15. (B) Representative Western blot analysis of ADAM15 expression in the HCC (T) and nontumor tissues (NT) was shown. (C) The data from the database UALCAN, GEPIA showed that ADAM15 was higher in HCC tissues compared to non-tumor tissues. (D) Data from UALCAN, GEPIA and Kaplan-Meier Plotter further demonstrated that high ADAM15 expression indicated poor survival of HCC patients. (E) The expressions of ADAM15 in human normal hepatocyte cell line L02 and HCC cell lines were examined using qRT-PCR. (F) Hep3B and HCCLM3 cells that were transfected with corresponding vectors were subjected to western blot for ADAM15 expression. Overexpression of ADAM15 promoted cell migration and invasion in Hep3B cells $(G)$, while down-regulation of ADAM15 inhibited cell migration and invasion in HCCLM3 cells $(H) . n=$ three independent experiments. ${ }^{*} P<0.05$.
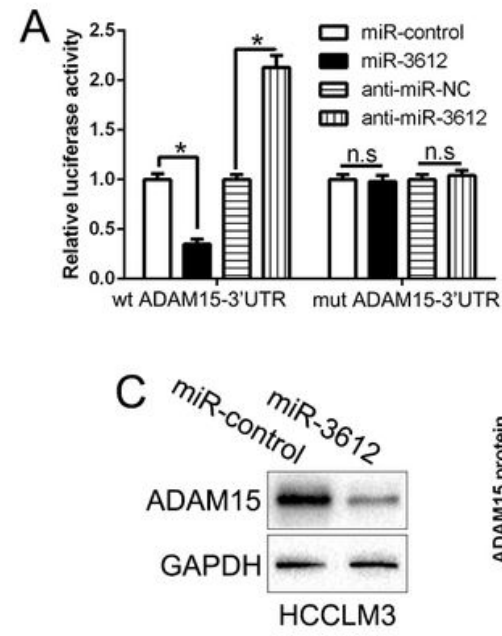

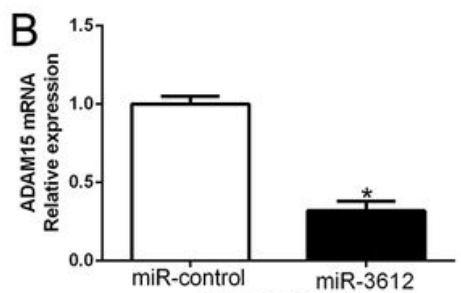

HCCLM3
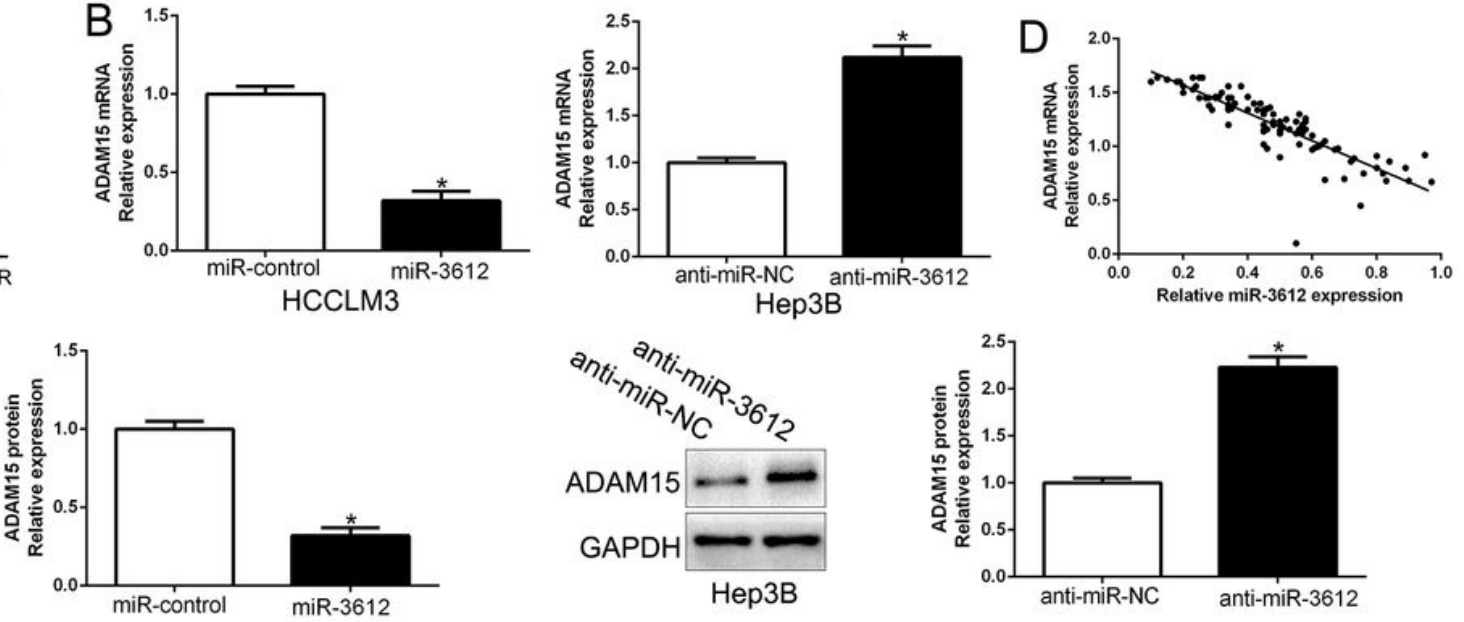

\section{Figure 6}

ADAM15 is identified as a direct target of miR-3612 in HCC. (A) miR-3612 significantly suppresses the luciferase activity that carried wild-type (wt) but not mutant (mt) 3'-UTR of ADAM15. Anti-miR-3612 led to a notable increase in the luciferase activity of wt 3'-UTR of ADAM15. (B) qRT-PCR analysis of ADAM15 mRNA expression in HCCLM3 cells with miR-3612 or miR-control vector transfection and Hep3B cells with anti-miR-3612 or anti-miR-NC vector transfection. (C) Overexpression of miR-3612 reduced the expression of ADAM15 protein in HCCLM3 cells and knockdown of miR-3612 increases the level of ADAM15 protein in Hep3B cells. (D) A significant inverse correlation between the mRNA levels of ADAM15 and miR-3612 was observed in HCC tissues. ${ }^{*} \mathrm{P}<0.05$. 

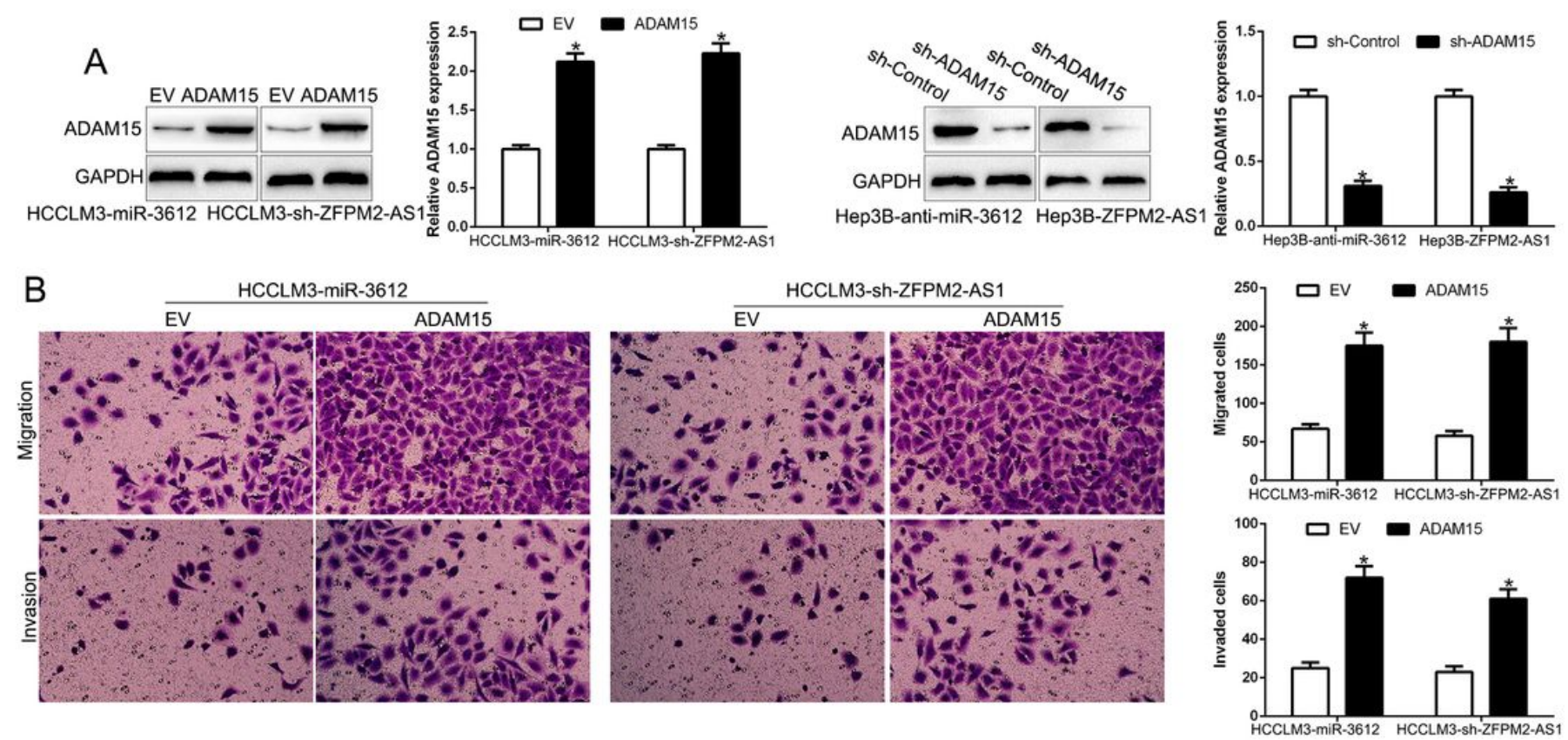

C
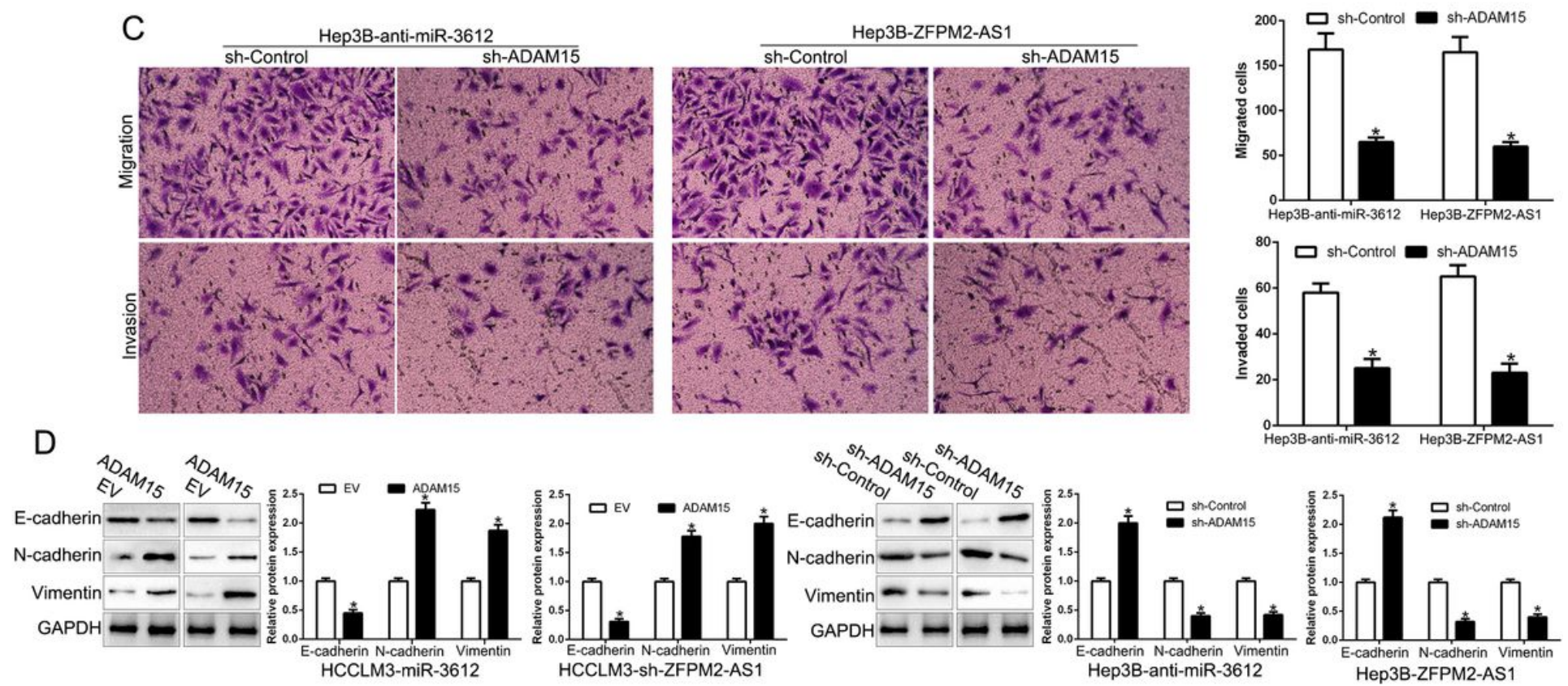

Figure 7

Modulation of ADAM15 partially abolishes ZFPM2-AS1 or miR-3612-mediated cellular processes in HCC. (A) miR-3612-overexpressing or ZFPM2-AS1-suppressive HCCLM3 cells that were transfected with empty vector (EV) or ADAM15 overexpression plasmid were subjected to western blot for ADAM15. miR-3612suppressive or ZFPM2-AS1-overexpressing Hep3B cells that were transfected with scrambled shRNA or ADAM15 shRNA were subjected to western blot for ADAM15. (B-D) ADAM15 restoration abrogated the effects of miR-3612 overexpression or ZFPM2-AS1 knockdown on cell migration, invasion and EMT of HCCLM3 cells. ADAM15 knockdown reversed the promotive effects of miR-3612 knockdown or ZFPM2AS1 overexpression in Hep3B cells. ${ }^{*} \mathrm{P}<0.05$. 

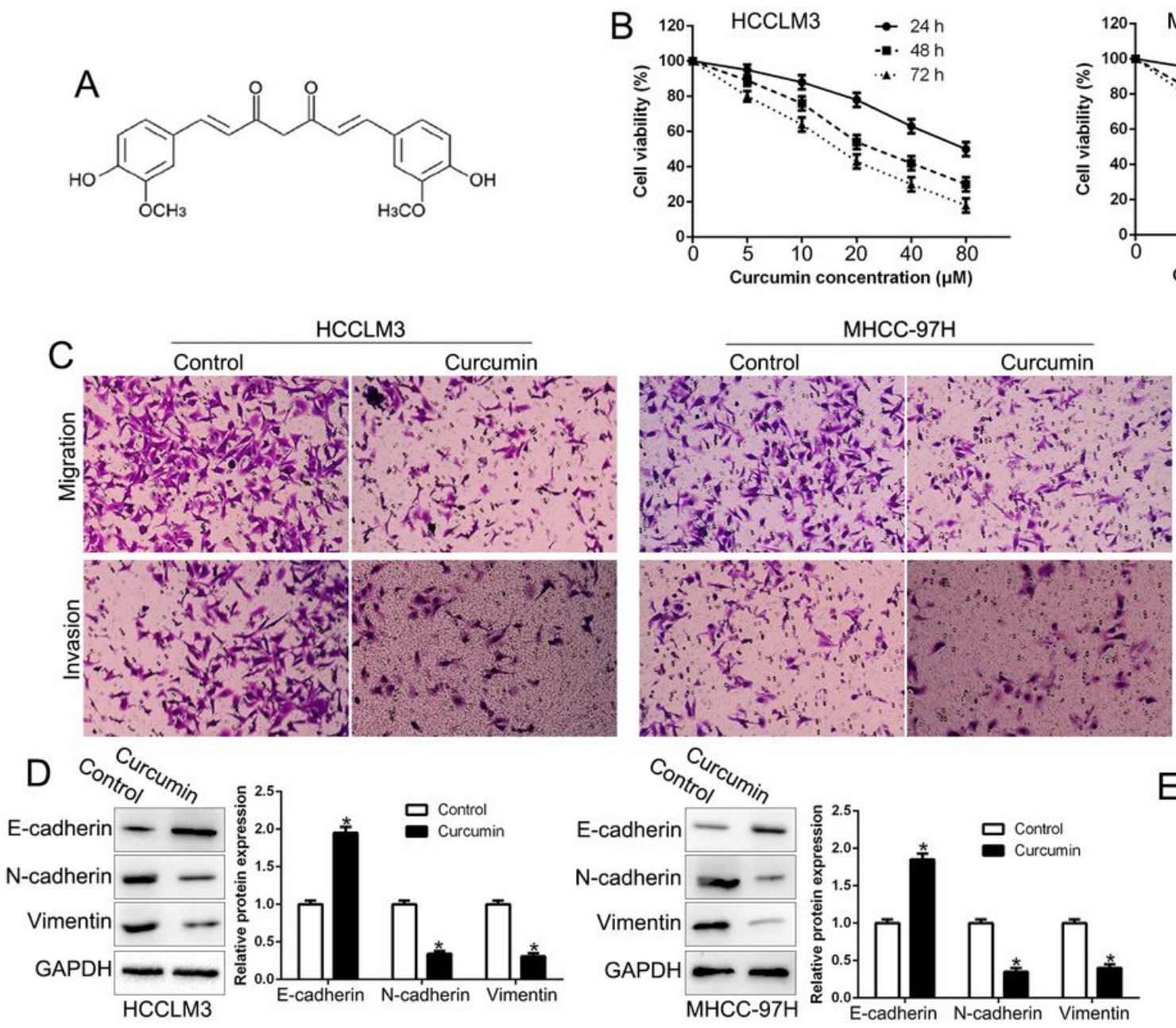

E
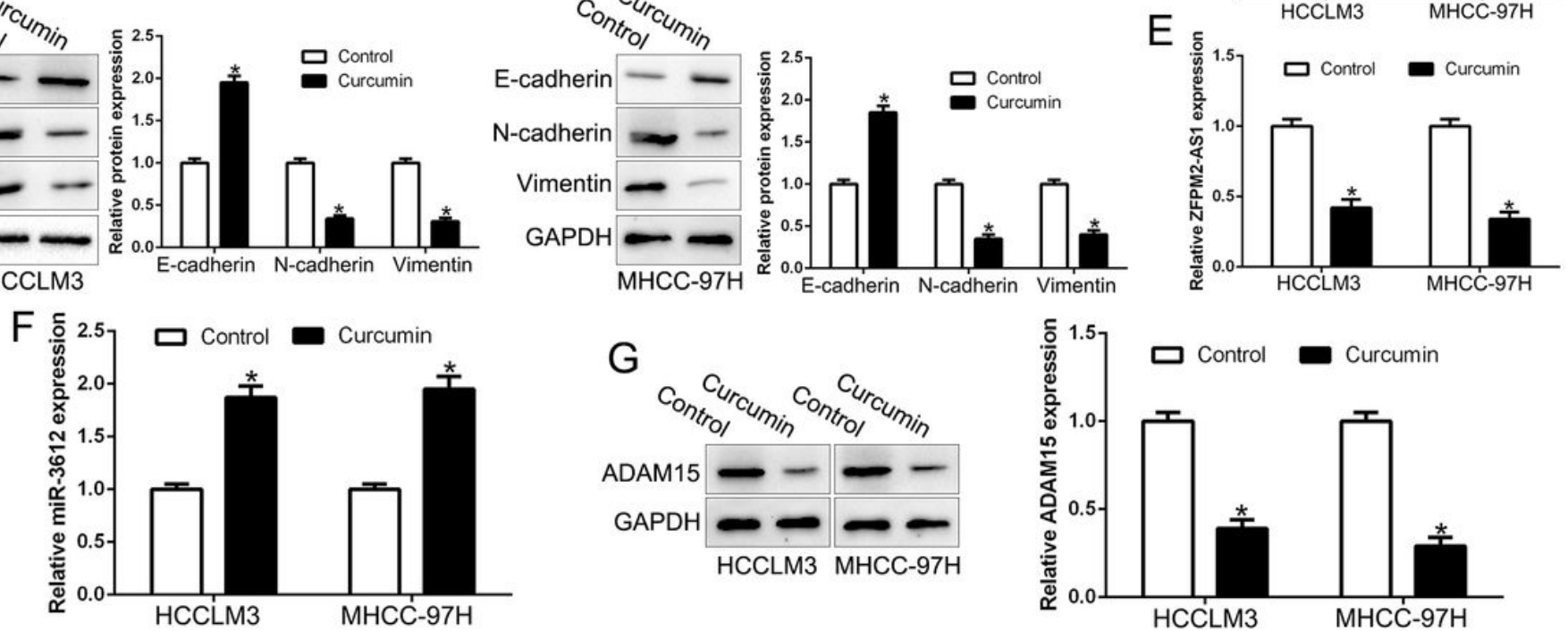
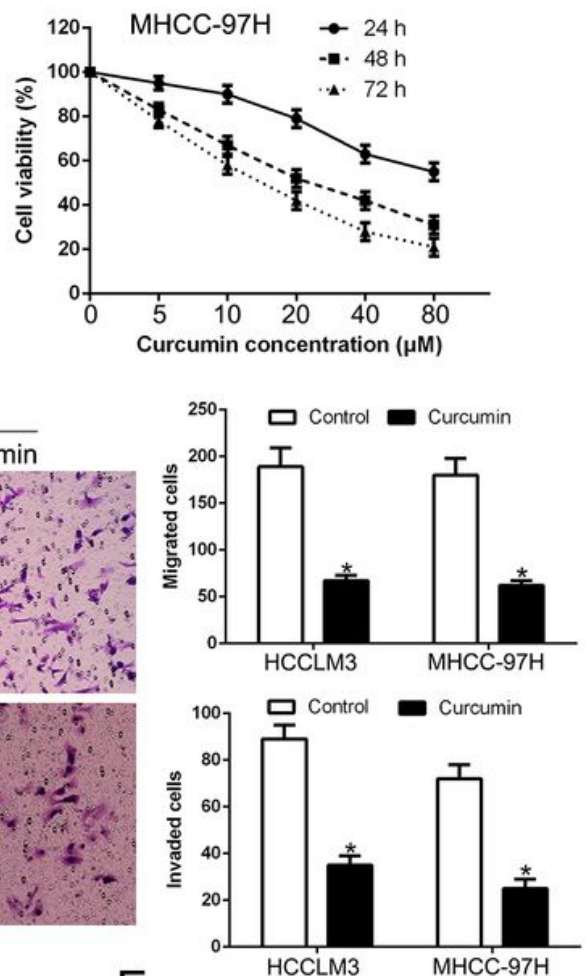
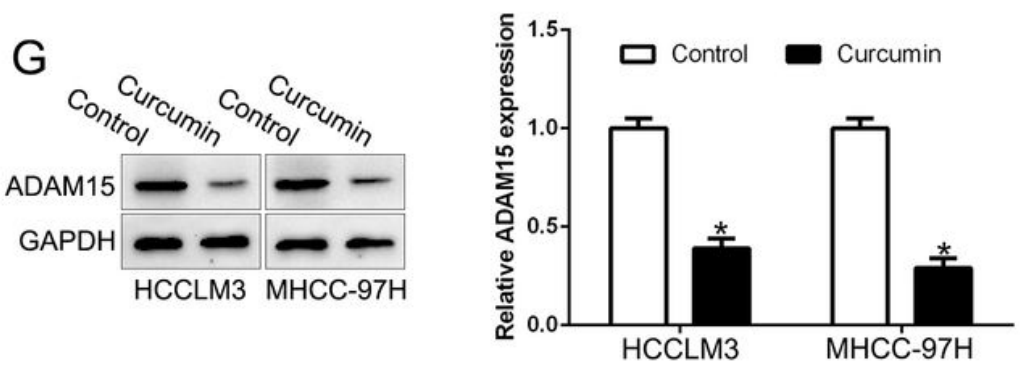

\section{Figure 8}

Curcumin attenuates HCC migration and invasion via ZFPM2-AS1/miR-3612/ADAM15 signaling pathway. (A) Chemical structure of curcumin. (B) Effect of curcumin on viability of HCCLM3 and MHCC97H for $24 \mathrm{~h}, 48 \mathrm{~h}$ and $72 \mathrm{~h}$. (C-D) Curcumin inhibited cell migration, invasion and EMT in HCCLM3 and MHCC-97H cells. Curcumin inhibited ZFPM2-AS1 (E) and ADAM15 (G) expression while increased miR3612 expression (F) in HCCLM3 and MHCC-97H cells. ${ }^{*} \mathrm{P}<0.05$. 